\title{
A new modified differential evolution algorithm scheme-based linear frequency modulation radar signal de-noising
}

\begin{abstract}
The main intention of this study was to investigate the development of a new optimization technique based on the differential evolution (DE) algorithm, for the purpose of linear frequency modulation radar signal de-noising. As the standard DE algorithm is a fixed length optimizer, it is not suitable for solving signal de-noising problems that call for variability. A modified crossover scheme called rand-length crossover was designed to fit the proposed variable-length $\mathrm{DE}$, and the new $\mathrm{DE}$ algorithm is referred to as the random variable-length crossover differential evolution (rvlx-DE) algorithm. The measurement results demonstrate a highly efficient capability for target detection in terms of frequency response and peak forming that was isolated from noise distortion. The modified method showed significant improvements in performance over traditional de-noising techniques.
\end{abstract}

Keyword: Chirp pulse generators; Differential evolution (DE); Target detection; Radar signal de-noising 